\title{
Genome-wide identification and evolutionary analyses of the $P P 2 C$ gene family with their expression profiling in response to multiple stresses in Brachypodium distachyon
}

\author{
Jianmei Cao ${ }^{1}$, Min Jiang ${ }^{1}$, Peng $\mathrm{Li}^{1}$ and Zhaoqing $\mathrm{Chu}^{1,2^{*}}$
}

\begin{abstract}
Background: The type- $2 \mathrm{C}$ protein phosphatases (PP2Cs), negatively regulating ABA responses and MAPK cascade pathways, play important roles in stress signal transduction in plants. Brachypodium distachyon is a new model plant for exploring the functional genomics of temperate grasses, cereals and biofuel crops. To date, genome-wide identification and analysis of the PP2C gene family in B. distachyon have not been investigated.
\end{abstract}

Results: In this study, 86 PP2C genes in B. distachyon were identified. Domain-based analyses of PP2C proteins showed that they all contained the phosphatase domains featured as 11 conserved signature motifs. Although not all phosphatase domains of BdPP2C members included all 11 motifs, tertiary structure analysis showed that four residues contributing to magnesium/manganese ions $\left(\mathrm{Mg}^{2+} / \mathrm{Mn}^{2+}\right)$ coordination were conserved, except for two noncanonical members. The analysis of their chromosomal localizations showed that most of the BdPP2C genes were located within the low CpG density region. Phylogenetic tree and synteny blocks analyses among B. distachyon, Arabidopsis thaliana and Oryza sativa revealed that all PP2C members from the three species can be phylogenetically categorized into 13 subgroups (A-M) and BdPP2Cs were evolutionarily more closely related to OSPP2CS than to AtPP2CS. Segmental duplications contributed particularly to the expansion of the $B d P P 2 C$ gene family and all duplicated BdPP2Cs evolved mainly from purifying selection. Real-time quantitative reverse transcription PCR (qRT-PCR) analysis showed that BdPP2Cs were broadly expressed in disparate tissues. We also found that almost all members displayed up-regulation in response to abiotic stresses such as cold, heat, PEG and NaCl treatments, but down-regulation to biotic stresses such as Ph14, Guy11 and F0968 infection.

Conclusions: In the present study, a comprehensive analysis of genome-wide identification and characterization of protein domains, phylogenetic relationship, gene and protein structure, chromosome location and expression pattern of the PP2C gene family was carried out for the first time in a new model monocot, i.e., B. distachyon. Our results provide a reference for genome-wide identification of the $P P 2 C$ gene family of other species and also provide a foundation for future functional research on PP2C genes in B. distachyon.

Keywords: B. distachyon, Protein phosphatases 2C (PP2C), Phylogenetic analysis, Gene expression profiling

\footnotetext{
* Correspondence: zqchu@sibs.ac.cn

${ }^{1}$ Shanghai Key Laboratory of Plant Functional Genomics and Resources,

Shanghai Chenshan Botanical Garden, Shanghai 201602, China

${ }^{2}$ Shanghai Chenshan Plant Science Research Center, Chinese Academy of

Sciences, Shanghai 201602, China
}

(c) 2016 Cao et al. Open Access This article is distributed under the terms of the Creative Commons Attribution 4.0 International License (http://creativecommons.org/licenses/by/4.0/), which permits unrestricted use, distribution, and reproduction in any medium, provided you give appropriate credit to the original author(s) and the source, provide a link to the Creative Commons license, and indicate if changes were made. The Creative Commons Public Domain Dedication waiver (http://creativecommons.org/publicdomain/zero/1.0/) applies to the data made available in this article, unless otherwise stated. 


\section{Background}

Wild plants are usually consistently exposed to various environmental challenges such as drought, high salinity, extreme temperature, heavy metals and pathogen infections, which affect their growth and development. To adapt to these unpredictable environmental stresses, plants have evolved signaling mechanisms to transmit stimuli to different cellular compartments and then respond to these stresses. Accumulating evidence indicates that reversible protein phosphorylation catalyzed by protein kinases and phosphatases plays important roles in cellular stresses signal transduction in plants [1]. In the past decades, several protein kinases have been extensively investigated and proved to be positive regulation factors responding to a diversity of abiotic and biotic stresses [2-5]. In contrast to the protein kinases, protein phosphatases are not well investigated.

Protein phosphatases modify protein function by removing the phosphate group from phosphorylated proteins. They are divided into three major classes: tyrosine phosphatases, serine/threonine phosphatases and dual-specificity phosphatases (DSPTPs). According to distinct amino acid sequences, different dependencies on metal ions and sensitivities to inhibitors such as cyclosporine A and okadaic acid, protein serine/threonine phosphatases can be classified into the phosphor-protein phosphatases (PPP), phosphoprotein metallophosphatases (PPM) and aspartate-based protein phosphatases [6]. The PPP family includes type 1 (PP1), type 2A (PP2A), type 4 (PP4), type 5 (PP5), type 6 (PP6), type 7 (PP7) and type 2B (PP2B), whereas the PPM family is represented by the $\mathrm{Mg}^{2+}$ - or $\mathrm{Mn}^{2+}$ - dependent type-2C protein phosphatases (PP2Cs) [7].

PP2Cs, evolutionarily conserved and found in archaea, bacteria, fungi, plants and animals, are implicated in regulating stress-signaling pathways and act as negative modulators of protein kinase cascades activated by diverse stresses. For instance, a PP2C phosphatase, RsbP, in Bacillus subtilis dephosphorylates RsbV (an anti-anti- $\sigma$ factor) and mediates the regulation during energy stress [8]. In Saccharomyces cerevisiae, PP2Cs play a negative role in the signal-transduction process responding to osmotic stress by counteracting the mitogen-activated protein kinase (MAPK) pathway [9]. Furthermore, one of the human PP2Cs was shown to inactivate the AMP-activated protein kinase (AMPK), a central component of the protein kinase cascade activated by ATP depletion [10]. In higher plants such as Arabidopsis, PP2C genes were demonstrated to regulate signaling pathways negatively by opposing the action of particular protein kinases. At least eight PP2Cs from subgroup A in Arabidopsis have been characterized as key factors in ABA signaling transduction. Briefly, subgroup A PP2Cs inactivate SnRK2 via dephosphorylation, and this inactivation is inhibited by ABA receptors, PYR/
PYL/RCRA, in an ABA-dependent manner [11]. The subgroup B PP2C, AP2C1, interacts with MPK4 or MPK6 and subsequently suppresses MAPK activities during wounding as well as pathogen stresses [12]. The subgroup C PP2C POL or PLL1 interacts with the receptor kinase CLV1, inducing and maintaining stem cell polarity $[13,14]$. The subgroup E PP2C AtPP2C6-6 interacts with Arabidopsis histone acetyl transferase GCN5 and controls the activation of stress-responsive genes in the stomatal signaling network [15-17]. The subgroup F PP2C WIN2 may interact with the bacterial effector HopW1-1 and regulate HopW1-1-induced plant resistance [18]. The unclustered PP2C KAPP interacts with different receptor-like protein kinases (RLKs) and is predicted to control plant immunity responses or hormone signaling $[19,20]$. To date, there are 80 and 90 genes coding for PP2C proteins identified using bioinformatics surveys in Arabidopsis and rice, respectively [21-24]. However, few $P P 2 C$ genes in monocots have been functionally investigated.

$B$. distachyon is a new model monocot for exploring the functional genomics of temperate grasses, cereals and biofuel crops. In 2010, the complete genome of the $B$. distachyon $\mathrm{Bd} 21$ was sequenced and the sequence data can be easily obtained via the Brachypodium Genome Resource (http://www.brachypodium.org) [25]. To our knowledge, a genome-wide analysis of the $P P 2 C$ gene family in $B$. distachyon has not been reported so far. Here, we first identified 86 PP2Cs based on phosphatase domains analysis. Then, we further examined whether all phosphatase domains of BdPP2C members harbored magnesium/manganese ions $\left(\mathrm{Mg}^{2+} / \mathrm{Mn}^{2+}\right)$ coordination residues through protein structural analysis. We also investigated $B d P P 2 C$ genes chromosomal localization, constructed the phylogenetic tree of all BdPP2C genes based on their PP2C domains and categorized them into 13 subgroups. Subsequently, we analyzed the duplication events contributing to the expansion and functional divergences of the $B d P P 2 C$ gene family. In addition, we examined the expression profiles of $B d P P 2 C$ genes in different tissues and their responses to different phytohormone treatments as well as various abiotic and biotic stresses. Our results provide a foundation for future functional analysis of the $P P 2 C$ gene family in stress responses in $B$. distachyon.

\section{Results and discussion}

\section{Genome-wide identification and characterization of BdPP2C genes}

Protein phosphatase PP2Cs are evolutionarily conserved [26]. During the evolution from prokaryotes to multicellular eukaryotes, the number of $P P 2 C$ genes increased from one member to as many as 130 members. In previous reports, one $\mathrm{PP} 2 \mathrm{C}$ member in Thermococcus, three 
in Synechocaccus, four in Bacillus, seven in Saccharomyces, 34 in Chlamydomonas, 51 in Physcomitrella, 57 in Selaginella, 80 in Arabidopsis, 90 in Oryza and 130 in Zea mays were characterized [26]. The increase and expansion of $P P 2 C$ genes from Archaea to higher plants may correlate with adaptations to complex environmental conditions for plants. To identify $P P 2 C$ candidate genes in $B$. distachyon, the InterPro PP2C domain "IPR001932" was used to search the Plaza2.5 database (http:// bioinformatics.psb.ugent.be/plaza/versions/plaza_v2_5/) and 88 putative $P P 2 C$ genes were found. By using Pfam and SMART domain identification tools, we found that 2 of the 88 putative PP2C genes lacked PP2C catalytic domains. Therefore, 86 genes in B. distachyon were identified as $P P 2 C$ family members. The $86 B d P P 2 C$ genes identified in this study encode proteins varying from 281 to 1087 amino acids in length, with large variations in isoelectric point $(\mathrm{pI})$ values from 4.24 to 9.31 and molecular weight from $30 \mathrm{kDa}$ to $120 \mathrm{kDa}$. The subcellular localization prediction indicated that most of the BdPP2C proteins might be located in cytoplasm, chloroplast or nucleus, while only a few might be located in cytoskeleton or mitochondria (Table 1). A total of 86 PP2C genes were anchored to corresponding chromosomes and designated as $\mathrm{BdPP2C1-}$ $B d P P 2 C 86$ according to their order on the chromosomes.

The PP2C phosphatase catalytic domain has been reported to harbor 11 conserved motifs, in which 4 conserved residues contribute to $\mathrm{Mg}^{2+} / \mathrm{Mn}^{2+}$ coordination [27, 28]. So we further checked if BdPP2Cs' phosphatase catalytic domain harbored 11 conserved motifs. The result obtained from multiple alignments of the 86 BdPP2C domains indicated that not all of the BdPP2C members contained all of the 11 conserved motifs. For example, Bradi1g54110, Bradi3g03870, Bradi3g43440 and Bradi2g27977 were found to be partial deletions in the C-terminal of PP2C phosphatase catalytic domain, thus probably resulting in elimination of a few important motifs and loss of functions. Further analysis showed that scattered amino acid residues also existed among conserved motifs in several BdPP2C members, which suggests that the amino acid composition of plant PP2C domains is quite complex (Additional file 1: Figure S1). In addition, the 3-D structure prediction of 86 $\mathrm{BdPP} 2 \mathrm{C}$ domains also showed the diversities of $\mathrm{PP} 2 \mathrm{C}$ proteins (Additional file 2: Figure S2). However, as shown in Additional file 1: Figure S1, the residues contributing to $\mathrm{Mg}^{2+} / \mathrm{Mn}^{2+}$ coordination in BdPP2Cs are absolutely conserved. The conserved amino acid residues [xxD], [DGxxG], [DG] and [GxxDN] (D, aspartic acid; G, glycine; $\mathrm{N}$, asparagine) were found within motifs $1,2,8$ and 11, respectively. The predicted protein tertiary structures further revealed that these 4 conserved residues were spatially clustered together in 84 BdPP2Cs (designated as canonical BdPP2Cs), except Bradi1g54080 and Bradilg54110, which were designated as noncanonical members (Fig. 1, Additional file 3: Figure S3).

\section{Phylogenetic, gene structural and protein domain analyses of BdPP2C}

To investigate the phylogenetic relationships of $P P 2 C$ genes between $B$. distachyon and other plants, we constructed a phylogenetic tree based on the alignments of PP2C domains in B. distachyon, Arabidopsis and rice using the maximum likelihood (ML) method. The phylogenetic analyses indicated that the $80 \mathrm{BdPP} 2 \mathrm{C}$ proteins were divided into 12 subfamilies $(\mathrm{A}-\mathrm{L})$, which is consistent with the PP2C groups found in Arabidopsis and rice. Each subfamily tree includes $\mathrm{PP} 2 \mathrm{C}$ protein from $B$. distachyon, Arabidopsis and rice (Fig. 2). Not surprisingly, the $\mathrm{BdPP} 2 \mathrm{C}$ clustered together with those from rice because both $B$. distachyon and rice are monocots, while AtPP2C tended to form independent branches. Furthermore, the distributions of PP2Cs in each subfamily were similar among B. distachyon, Arabidopsis and rice except for the subgroups I and $\mathrm{K}$ (Table 2), which suggests that the $P P 2 C$ gene family has evolved from one common ancestor in the three plants. The other three members, i.e., Bradilg04540, Bradilg54110 and Bradilg54080, grouped into a new clade, $M$. In addition, the remaining three members, i.e., Bradi2g40550, Bradi4g27880 and Bradi2g40950, did not cluster with any other group, and each of them formed a single branch (Fig. 2).

To determine the phylogenetic relationships among the $\mathrm{BdPP} 2 \mathrm{C}$ proteins, we constructed a phylogenetic tree from the alignments of the $86 \mathrm{PP} 2 \mathrm{C}$ domains identified above. As shown in Fig. 2 and Fig. 3a, all of the BdPP2C proteins still remained in the same subfamily. Because the pattern diversity of exon/intron structure and protein domain often plays an important role in the evolution of gene families, the patterns of exon/intron structure of $B d P P 2 C$ genes and conserved domains were examined according to their phylogenetic relationships. The investigation of exon/intron structures revealed that the majority of members in the same subfamily shared similar exon numbers and different exon and intron lengths. However, three members in the $M$ group, consisting of 5, 13 and 17 exons, were largely distinct in exon/intron arrangements, although their phylogenetic relationship was supported by $100 \%$ bootstrap value. More interestingly, almost all BdPP2Cs lacking introns were members of the $\mathrm{K}$ subgroup, and the number of $\mathrm{PP} 2 \mathrm{C}$ members in the $\mathrm{K}$ subgroup in $B$. distachyon was four or two times more than that in Arabidopsis and rice, respectively (Fig. 3b, Table 2). Previous studies suggested that genes lacking introns would rapidly evolve via gene duplication events [29-31]. Therefore, the 
Table 1 List of 86 BdPP2C genes and their basic characterizations

\begin{tabular}{|c|c|c|c|c|c|c|}
\hline Gene identifier & Gene name & Size (aa) & Mass (kDa) & $\mathrm{pl}$ & Subcellular localization & Subgroup \\
\hline Bradi1g02920 & $\mathrm{BdPP} 2 \mathrm{C} 1$ & 383 & 41.607 & 6.7 & cyto & D \\
\hline Bradi1g03690 & $\mathrm{BdPP} 2 \mathrm{C2}$ & 662 & 71.157 & 5.62 & chlo & C \\
\hline Bradi1g04520 & $\mathrm{BdPP} 2 \mathrm{C} 3$ & 474 & 50.021 & 8.36 & chlo & K \\
\hline Bradi1g04540 & $\mathrm{BdPP} 2 \mathrm{C} 4$ & 566 & 61.298 & 6.36 & chlo & M \\
\hline Bradi1g07870 & $\mathrm{BdPP} 2 \mathrm{C} 5$ & 379 & 41.509 & 9.31 & chlo & D \\
\hline Bradi1g16630 & BdPP2C6 & 381 & 40.055 & 8.83 & chlo & $B$ \\
\hline Bradi1g16810 & $\mathrm{BdPP} 2 \mathrm{C} 7$ & 432 & 46.273 & 7.5 & chlo & $\mathrm{H}$ \\
\hline Bradi1g19620 & $\mathrm{BdPP} 2 \mathrm{C} 8$ & 476 & 49.814 & 5.29 & cyto & L \\
\hline Bradi1g24400 & BdPP2C9 & 428 & 46.447 & 5.83 & nucl & $\mathrm{E}$ \\
\hline Bradi1g26690 & $\mathrm{BdPP} 2 \mathrm{C} 10$ & 290 & 31.918 & 6.01 & cyto & $\mathrm{F}$ \\
\hline Bradi1g30200 & BdPP2C11 & 392 & 43.45 & 8.77 & mito & $\mathrm{D}$ \\
\hline Bradi1g31080 & BdPP2C12 & 366 & 40.344 & 4.94 & nucl & I \\
\hline Bradilg33900 & $\mathrm{BdPP} 2 \mathrm{C} 13$ & 281 & 30.172 & 5.01 & cyto & $\mathrm{F}$ \\
\hline Bradi1g36330 & BdPP2C14 & 309 & 33.654 & 6.25 & cyto & K \\
\hline Bradi1g36690 & BdPP2C15 & 309 & 33.423 & 5.51 & cyto & K \\
\hline Bradi1g36920 & BdPP2C16 & 359 & 38.823 & 4.85 & cyto & G \\
\hline Bradi1g37500 & $\mathrm{BdPP} 2 \mathrm{C} 17$ & 312 & 33.409 & 6.33 & chlo & K \\
\hline Bradi1g37530 & BdPP2C18 & 312 & 33.409 & 6.33 & chlo & $\mathrm{K}$ \\
\hline Bradi1g38670 & BdPP2C19 & 362 & 38.975 & 8.9 & chlo & $\mathrm{F}$ \\
\hline Bradi1g47710 & BdPP2C20 & 353 & 38.882 & 5.01 & cyto & G \\
\hline Bradi1g54080 & BdPP2C21 & 263 & 29.067 & 7.75 & extr & M \\
\hline Bradi1g54110 & BdPP2C22 & 690 & 75.85 & 7.81 & chlo & M \\
\hline Bradi1g60520 & BdPP2C23 & 450 & 48.306 & 5.45 & chlo & $\mathrm{H}$ \\
\hline Bradi1g64780 & BdPP2C24 & 433 & 46.283 & 7.02 & chlo & $\mathrm{H}$ \\
\hline Bradi1g65520 & BdPP2C25 & 387 & 40.918 & 6.63 & chlo & B \\
\hline Bradi1g66650 & BdPP2C26 & 625 & 68.08 & 6.37 & nucl & C \\
\hline Bradi1g66920 & BdPP2C27 & 405 & 43.564 & 5.59 & nucl & A \\
\hline Bradi1g70680 & BdPP2C28 & 403 & 43.323 & 8.57 & chlo & $\mathrm{D}$ \\
\hline Bradi1g71690 & BdPP2C29 & 582 & 60.563 & 4.28 & chlo & K \\
\hline Bradi1g75940 & BdPP2C30 & 399 & 44.021 & 9.12 & nucl & $\mathrm{D}$ \\
\hline Bradi2g03970 & BdPP2C31 & 321 & 34.228 & 4.95 & chlo & K \\
\hline Bradi2g11350 & BdPP2C32 & 386 & 42.481 & 4.81 & cyto & G \\
\hline Bradi2g13820 & BdPP2C33 & 470 & 50.126 & 8.81 & nucl & $\mathrm{H}$ \\
\hline Bradi2g14420 & BdPP2C34 & 424 & 44.985 & 5.35 & nucl & A \\
\hline Bradi2g14740 & BdPP2C35 & 503 & 54.522 & 4.67 & nucl & $\mathrm{F}$ \\
\hline Bradi2g15840 & BdPP2C36 & 385 & 41.206 & 5.66 & nucl & A \\
\hline Bradi2g18510 & BdPP2C37 & 374 & 40.455 & 5.63 & chlo & A \\
\hline Bradi2g27970 & BdPP2C38 & 340 & 37.233 & 7.5 & chlo & E \\
\hline Bradi2g27977 & BdPP2C39 & 315 & 34.512 & 5.3 & chlo & E \\
\hline Bradi2g38640 & $\mathrm{BdPP} 2 \mathrm{C} 40$ & 385 & 41.564 & 4.69 & cyto & G \\
\hline Bradi2g38770 & BdPP2C41 & 546 & 59.207 & 5.74 & chlo & C \\
\hline Bradi2g40550 & BdPP2C42 & 656 & 73.068 & 5.96 & cyto & 1 \\
\hline Bradi2g40950 & BdPP2C43 & 390 & 42.049 & 6.41 & chlo & M \\
\hline
\end{tabular}


Table 1 List of 86 BdPP2C genes and their basic characterizations (Continued)

\begin{tabular}{|c|c|c|c|c|c|c|}
\hline Bradi2g41950 & BdPP2C44 & 480 & 49.799 & 4.87 & cyto & A \\
\hline Bradi2g43700 & BdPP2C45 & 289 & 31.763 & 4.89 & cysk & $\mathrm{F}$ \\
\hline Bradi2g45470 & BdPP2C46 & 392 & 42.104 & 5.47 & chlo & A \\
\hline Bradi2g54810 & BdPP2C47 & 403 & 42.912 & 6.06 & nucl & A \\
\hline Bradi2g62650 & BdPP2C48 & 384 & 42.197 & 8.56 & mito & D \\
\hline Bradi3g03480 & BdPP2C49 & 380 & 39.678 & 5.3 & chlo & K \\
\hline Bradi3g03870 & $\mathrm{BdPP} 2 \mathrm{C} 50$ & 289 & 30.628 & 5.32 & chlo & $\mathrm{F}$ \\
\hline Bradi3g05990 & BdPP2C51 & 372 & 40.923 & 5.14 & nucl & I \\
\hline Bradi3g08390 & BdPP2C52 & 366 & 38.917 & 5.86 & chlo & E \\
\hline Bradi3g09560 & BdPP2C53 & 361 & 38.717 & 6.68 & chlo & $\mathrm{F}$ \\
\hline Bradi3g10350 & BdPP2C54 & 1087 & 120.827 & 5.05 & chlo & L \\
\hline Bradi3g18090 & BdPP2C55 & 318 & 34.579 & 7.15 & chlo & $\mathrm{F}$ \\
\hline Bradi3g25590 & BdPP2C56 & 282 & 29.87 & 4.59 & cyto & K \\
\hline Bradi3g32240 & BdPP2C57 & 402 & 43.492 & 6.36 & nucl & G \\
\hline Bradi3g32480 & BdPP2C58 & 395 & 43.639 & 8.25 & chlo & D \\
\hline Bradi3g39540 & BdPP2C59 & 525 & 56.595 & 5.06 & chlo & E \\
\hline Bradi3g43360 & $\mathrm{BdPP} 2 \mathrm{C} 60$ & 521 & 55.581 & 7.6 & chlo & $E$ \\
\hline Bradi3g43430 & BdPP2C61 & 418 & 45.232 & 5.13 & nucl & I \\
\hline Bradi3g43440 & BdPP2C62 & 290 & 31.948 & 5.66 & nucl & I \\
\hline Bradi3g46160 & BdPP2C63 & 268 & 28.242 & 5.58 & cyto & K \\
\hline Bradi3g46430 & BdPP2C64 & 452 & 48.558 & 5.87 & chlo & $\mathrm{H}$ \\
\hline Bradi3g48280 & BdPP2C65 & 378 & 41.453 & 5.48 & mito & 1 \\
\hline Bradi3g49540 & BdPP2C66 & 365 & 38.711 & 5.59 & cyto & K \\
\hline Bradi3g 49550 & BdPP2C67 & 324 & 36.604 & 5.33 & cyto & K \\
\hline Bradi3g52110 & BdPP2C68 & 596 & 64.92 & 5.44 & chlo & C \\
\hline Bradi3g54290 & BdPP2C69 & 360 & 40.2 & 5.1 & cysk & G \\
\hline Bradi4g03520 & $\mathrm{BdPP} 2 \mathrm{C} 70$ & 390 & 42.584 & 9.2 & cyto & D \\
\hline Bradi4g15117 & BdPP2C71 & 1022 & 116.521 & 5.87 & cyto & J \\
\hline Bradi4g19660 & $\mathrm{BdPP} 2 \mathrm{C} 72$ & 438 & 47.799 & 6.24 & cyto & $E$ \\
\hline Bradi4g21510 & $\mathrm{BdPP} 2 \mathrm{C} 73$ & 378 & 40.097 & 5.94 & chlo & B \\
\hline Bradi4g27880 & $\mathrm{BdPP} 2 \mathrm{C} 74$ & 360 & 39.49 & 7.14 & nucl & 1 \\
\hline Bradi4g28100 & BdPP2C75 & 363 & 38.537 & 5.79 & nucl & A \\
\hline Bradi4g32230 & BdPP2C76 & 441 & 48.086 & 5.77 & chlo & $\mathrm{H}$ \\
\hline Bradi4g37710 & BdPP2C77 & 362 & 39.39 & 4.85 & nucl & 1 \\
\hline Bradi4g40490 & BdPP2C78 & 392 & 41.132 & 8.02 & chlo & B \\
\hline Bradi4g44750 & BdPP2C79 & 436 & 47.474 & 5.23 & cyto & E \\
\hline Bradi5g08830 & $\mathrm{BdPP} 2 \mathrm{C} 80$ & 519 & 57.198 & 4.94 & chlo & C \\
\hline Bradi5g11780 & BdPP2C81 & 444 & 47.535 & 4.24 & chlo & $\mathrm{H}$ \\
\hline Bradi5g11980 & BdPP2C82 & 282 & 30.706 & 6.26 & cyto & $\mathrm{F}$ \\
\hline Bradi5g14730 & BdPP2C83 & 431 & 47.974 & 4.38 & cyto & 1 \\
\hline Bradi5g19410 & BdPP2C84 & 393 & 43.381 & 6.33 & cyto & D \\
\hline Bradi5g21140 & BdPP2C85 & 316 & 34.51 & 8.6 & chlo & $\mathrm{F}$ \\
\hline Bradi5g24530 & BdPP2C86 & 284 & 30.813 & 4.87 & nucl & F \\
\hline
\end{tabular}

cyto cytoplasm, chlo chloroplast, nucl nucleus, mito mitochondria, extr extracellular, cysk cytoskeleton 


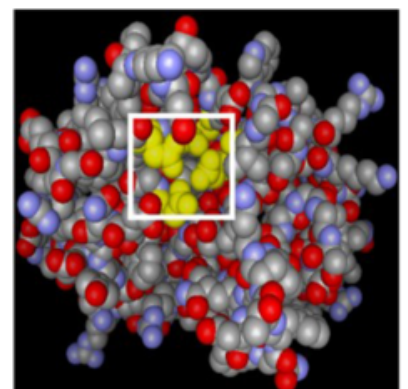

$\mathrm{BdPP} 2 \mathrm{Cs}$ (canonical PP2Cs)

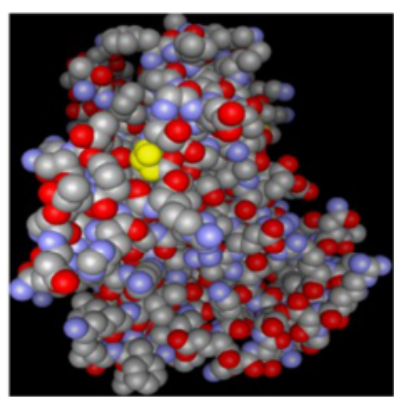

Bradi1g54080

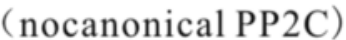

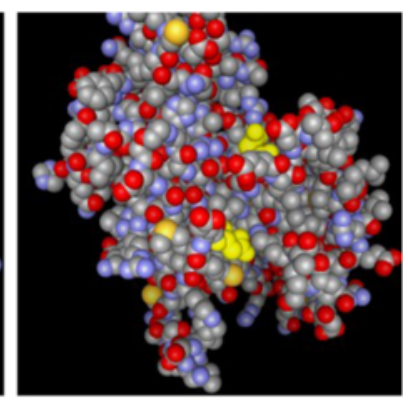

Bradilg54110

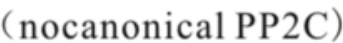

Fig. 1 The predicted tertiary structure of BdPP2C domains showing their conserved $\mathrm{Mg}^{2+} / \mathrm{Mn}^{2+}$ coordination sites in B. distachyon. The tertiary structures of BdPP2C domains were predicted using the Phyre 2 tool. The yellow regions indicated by white boxes represent conserved residues that contribute to $\mathrm{Mg}^{2+} / \mathrm{Mn}^{2+}$ coordination

expansion of the BdPP2C K subfamily may be associated with gene duplication events.

When analyzing the phosphatase domains, we found that other domains were associated with the main PP2C domain, including S-TKc (ser/thr kinase catalytic domain), FHA (forkhead associated domain) and CNB (cyclic nucleotide-binding domain). As shown in Fig. 3c, S-TKc was found in Bradi3g10350, Bradi4g15117 and Bradi2g40550; FHA was identified in Bradilg54110 and Bradilg04540; CNB was only present in Bradi3g10350. However, the transmembrane region identified in AtPP2Cs and OsPP2Cs was not found in any BdPP2Cs. The S-TKc or FHA domains have been found in several Arabidopsis and rice PP2C genes, including AT2G40860, Os01g36080 and Os11g37540 or At5G19280, Os03g59530 and Os07g11010. PP2Cs with FHA domain known as the KAPP (kinase-associated protein phosphatase) were reported to play important roles in interacting with RLK and have been characterized as the first downstream negative regulator of RLK in plant development in Arabidopsis [18]. Hence, it would be interesting to investigate the different biological functions of $B d P P 2 C$ s harboring these special domains.

\section{Chromosomal location and duplications of $B d P P 2 C$ genes}

To analyze the genomic distribution of $B d P P 2 C$ genes, we marked their approximate position on each chromosome based on the information obtained from the Brachypodium genome database. The results showed that all of the 86 BdPP2C genes were localized on five chromosomes and their distribution appeared to be uneven. Further analysis indicated that the number of $B d P P 2 C$ genes on each chromosome was proportional to the chromosome length. 30, 18, 21, 10 and $7 \mathrm{BdPP} 2 \mathrm{C}$ genes were mapped on chromosome 1, 2, 3, 4 and 5, respectively (Fig. 4). We also marked the densities of CpG islands on each chromosome. As shown in Fig. 4, the deeper color region on chromosomes represents the higher density of $\mathrm{CpG}$ islands; the gray or white region on chromosomes represents the lower density of CpG islands. We found that most of the $B d P P 2 C$ genes were located on gray or white regions, indicating that they might be transcriptionally active.

During evolutionary processes, large segmental duplications and small-scale tandem duplications are two major mechanisms to generate new genes, which contribute to the genome complexities in the plant kingdom [32]. Indeed, previous studies have reported that $P P 2 C$ gene families mainly expanded through whole-genome and chromosomal segment duplications, both in rice and Arabidopsis [23]. In B. distachyon, paralogous relationship analysis showed that six major chromosomal duplication events happened among the $B$. distachyon chromosomes, covering $92.1 \%$ of the whole genome [25]. Closely related genes located within a distance of less than $200 \mathrm{~kb}$ on the same chromosome are defined as tandem duplications, otherwise they are segmental duplications [33]. According to this principle, 22 pairs of paralogous $B d P P 2 C$ genes were found to be involved in segmental duplication events and no tandem duplication gene pairs were found in the $B d P P 2 C$ family. Analysis of these 22 pairs of segmental duplication $B d P P 2 C$ genes showed that 16 were located in 6 major duplicated chromosomal blocks (Fig. 5a). Amino acid alignment analysis indicated that two counterparts of each gene pair were from the same subgroup (Table 3). To determine the selection pressures for these duplicated $B d P P 2 C$ genes, we calculated the substitution ratio of non-synonymous (Ka) to synonymous (Ks) mutations for these 22 pairs of duplicated $B d P P 2 C$ genes. The ratio $\mathrm{Ka} / \mathrm{Ks}$ can be used to measure the selection acting among the duplicated gene pairs. Commonly, if the value of $\mathrm{Ka} / \mathrm{Ks}$ is less than 1 , the duplicated gene pairs may evolve from purifying selection (also called negative 


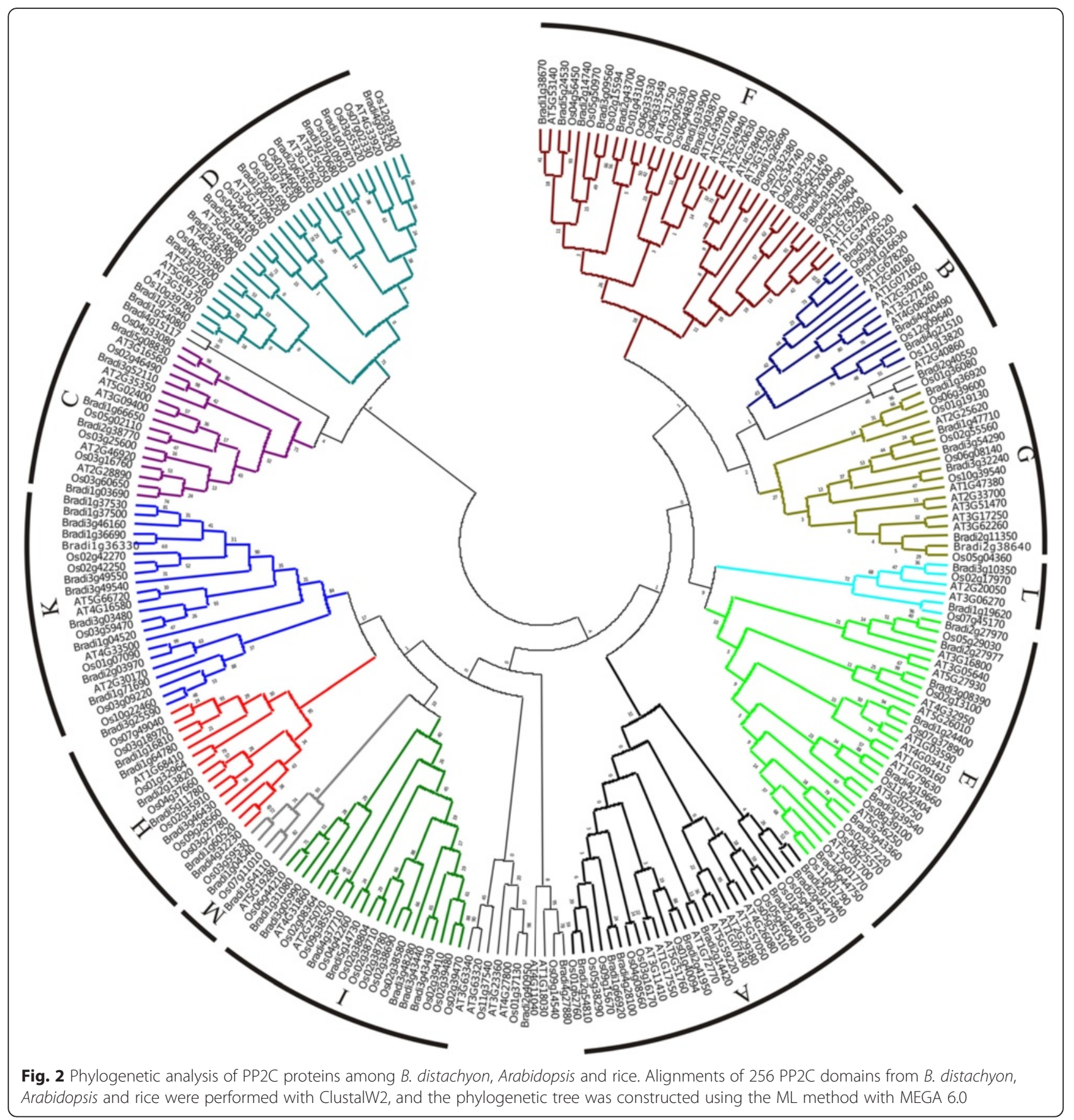

selection); $\mathrm{Ka} / \mathrm{Ks}=1$ means neutral selection; while $\mathrm{Ka} /$ Ks $>1$ means positive selection [34]. The result showed that $\mathrm{Ka} / \mathrm{Ks}$ for 22 pairs of duplicated $B d P P 2 C$ genes was less than 1, suggesting that all duplicated $B d P P 2 C$ genes have evolved mainly from purifying selection (Table 3 ). We also calculated the divergence time (as $\mathrm{T}=\mathrm{Ks} / 2 \lambda$ ) among 22 pairs of duplicated $B d P P 2 C$ genes based on a clock-like rate of $6.5 \times 10^{-9}$ mutations per synonymous site per year, as proposed previously [35]. The result in Table 3 showed that divergence events of duplicated
$B d P P 2 C$ genes were estimated to have occurred around 50.8-131.5 Mya (million years ago), and the majority of gene pairs diverged long before the divergence time of grass species (56-73 Mya) $[25,36]$. This finding is consistent with a previous report that genes involved in conserved signal transduction regulation pathways are preferentially retained [37], and this could explain why many $B d P P 2 C$ genes were retained during the long evolutionary history. In addition, we examined orthologous PP2C gene pairs between B. distachyon and Arabidopsis 
Table 2 The distribution of $P P 2 C$ genes in Arabidopsis, rice and B. distachyon

\begin{tabular}{llll}
\hline $\begin{array}{l}\text { Subgroup of } \\
\text { PP2C genes }\end{array}$ & $\begin{array}{l}\text { Numbers of } \\
\text { AtPP2Cs }\end{array}$ & $\begin{array}{l}\text { Numbers of } \\
\text { OsPP2Cs }\end{array}$ & $\begin{array}{l}\text { Numbers of } \\
\text { BdPP2Cs }\end{array}$ \\
\hline A & 10 & 10 & 8 \\
B & 6 & 3 & 4 \\
C & 7 & 6 & 5 \\
D & 9 & 11 & 9 \\
E & 12 & 12 & 8 \\
F & 13 & 11 & 11 \\
G & 6 & 8 & 6 \\
H & 3 & 7 & 7 \\
I & 2 & 12 & 7 \\
J & 2 & 1 & 1 \\
K & 3 & 6 & 12 \\
L & 2 & 0 & 2 \\
M & 0 & 0 & 3 \\
Single branch 1 & 1 & 1 & 1 \\
Single branch 2 & 1 & 1 & 1 \\
Single branch 3 & 2 & & 1 \\
\hline
\end{tabular}

and between $B$. distachyon and rice. As shown in Fig. 5b, $34 B d P P 2 C$ genes have one or two putative orthologs in rice; however, only seven $B d P P 2 C$ genes have orthologs in Arabidopsis, which suggests that the majority of $B d P P 2 C$ s' orthologs appeared after the divergence of monocots and dicots. Considering that gene orthologs often share similar functions [38], we infer that the functions of $B$. distachyon $P P 2 C$ genes have more similarity with rice.

\section{Expression profiles analysis of $B d P P 2 C$ genes in disparate} tissues and under various stress conditions

To investigate the expression profile of the $86 \mathrm{~B}$. distachyon $P P 2 C$ genes under normal growth conditions, we used quantitative reverse transcription-PCR (qRTPCR) analysis to examine their transcription levels in three different tissues: roots, stems and leaves. Most $B d P P 2 C$ genes were observed to have a very broad expression range, and barely detectable or no expression was observed for only nine genes (BdPP2C15, BdPP2C17, BdPP2C18, BdPP2C21, BdPP2C27, BdPP2C34, BdPP2C37, $B d P P 2 C 56$ and BdPP2C63). The qRT-PCR data showed that $13 B d P P 2 C$ genes were highly expressed in all three tissues, while 26 displayed weak expression. Seven $B d P P 2 C$ genes (BdPP2C24, BdPP2C25, BdPP2C53, BdPP2C73, $B d P P 2 C 74, B d P P 2 C 75$ and $B d P P 2 C 84)$ were found to be highly expressed preferentially in stems; and two (BdPP2C33 and BdPP2C39) were highly expressed in leaves. However, none of the $B d P P 2 C$ genes was highly preferentially expressed in roots (Fig. 6). The expression profiles data of $B d P P 2 C$ genes in various tissues are listed in Additional file 4: Table S3. We also compared the 22 duplicated $B d P P 2 C$ gene pairs and found that the expression pattern was very similar for 6 gene pairs, i.e., BdPP2C11/30, BdPP2C19/ 53, BdPP2C20/69, BdPP2C30/58, BdPP2C35/45 and $B d P P 2 C 55 / 85$. On the other hand, the remaining 15 pairs showed differential expression patterns. Recent studies suggested that there was a positive correlation of duplicated gene pairs between the degree of differential expression and their divergence time, which meant that the longer the duplication, the more obvious the differentiation of the expression [39-41]. In our analysis, we found that the divergence times for these seven duplicated gene pairs with similar expression pattern were actually more recent (50.8-86.9Mya). These results were also consistent with the expression of AtPP2C and OsPP2C paralogous gene pairs, indicating the diversification of expression between duplicated $P P 2 C$ gene pairs.

It has been demonstrated that subgroup A PP2Cs in Arabidopsis, foxtail millet and rice are transcriptionally upregulated upon exogenous $\mathrm{ABA}$ treatment or stress conditions that stimulate ABA biosynthesis [23, 24, 42, 43]. Seven members of subgroup A AtPP2Cs have been characterized as negative regulators of $\mathrm{ABA}$ responses in ABA-mediated physiological processes [44-49]. In $B$. distachyon, subgroup A $B d P P 2 C$ s includes eight members (BdPP2C27, BdPP2C34, BdPP2C36, BdPP2C37, $B d P P 2 C 44, B d P P 2 C 46, B d P P 2 C 47$ and BdPP2C75). The qRT-PCR result suggested that six of these genes were highly induced by exogenous ABA treatment, the exceptions being $B d P P 2 C 27$ and $B d P P 2 C 34$. Consistently, $B d P P 2 C$ s from subgroup A were also differentially upregulated by drought, salt, cold, heat or $\mathrm{H}_{2} \mathrm{O}_{2}$ treatment. Among them, $B d P P 2 C 36, B d P P 2 C 37$ and $B d P P 2 C 44$ were continuously up-regulated and remained at a high level upon all of these treatments. Both BdPP2C46 and $B d P P 2 C 47$ were up-regulated by drought, salt, cold, or $\mathrm{H}_{2} \mathrm{O}_{2}$, whereas they were down-regulated by heat treatment. For $B d P P 2 C 75$, the expression level increased under drought or salt treatment; however, cold, heat and $\mathrm{H}_{2} \mathrm{O}_{2}$ repressed its expression (Fig. 7). In addition, $B d P P 2 C 70$ from subgroup D, BdPP2C13 from subgroup $F$ and BdPP2C32 from subgroup $\mathrm{G}$ also exhibited strongly increased expression levels in response to $\mathrm{ABA}$ and abiotic treatments (Fig. 7), suggesting that they may participate in the regulation of ABA signaling pathways. Similarly, the expression profiles of $B d P P 2 C$ s in other subgroups under exogenous hormone or abiotic treatments for $3 \mathrm{~h}$ or $6 \mathrm{~h}$ were analyzed as well using qRTPCR. The other two exogenous hormones, i.e., MeJA and SA, were also tested. The heatmaps derived from expression profiles revealed that about $50-80 \%$ of the 


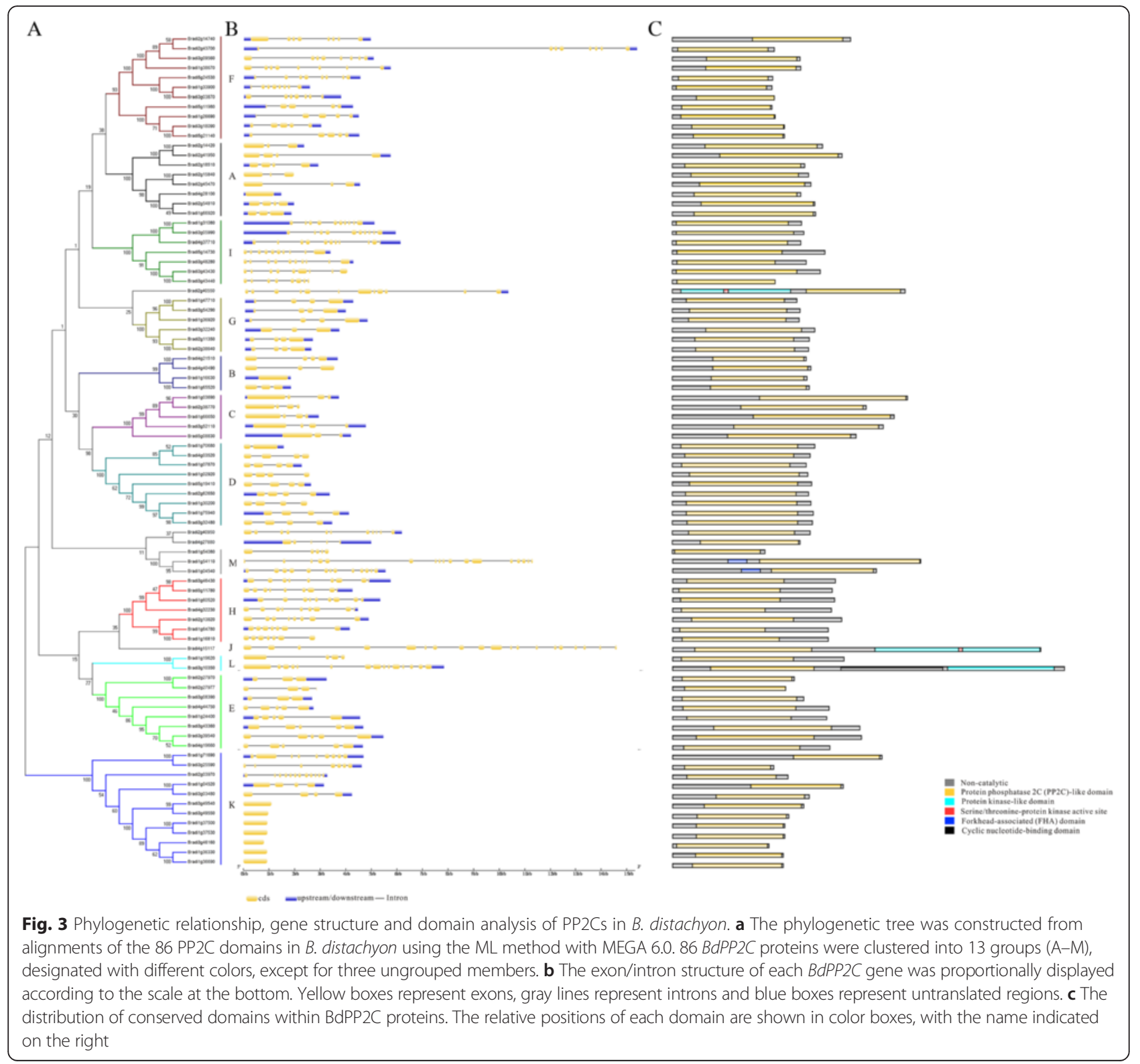

$B d P P 2 C$ genes were induced by various abiotic conditions or ABA treatment. On the other hand, most of the $B d P P 2 C$ genes were up-regulated within $3 \mathrm{~h}$ of treatment by MeJA or SA, whereas only 15 and $26 B d P P 2 C$ genes maintained the increase until $6 \mathrm{~h}$, respectively. In our analysis, the expression profiles of $B d P P 2 C$ genes under abiotic stresses, including cold, heat, drought and high salinity, showed differential and overlapping expression patterns. Previous studies have suggested that different stresses can activate the same genes in distinct signaling pathways $[50,51]$, which might be the result of the production of some common signaling component such as ABA or calcium triggered by different stress stimuli. Increasing these signaling components leads to activation of protein kinases, including MAPK, CDPK, CCaMK and
SnRK2, and then regulates the activities of transcription factors, leading to downstream responses. Moreover, such overlapping expression patterns might be the basis of crosstalk between different pathways, promoting the complex signal pathways for plants under abiotic stress conditions. Furthermore, we treated the $B$. distachyon seedlings with $\mathrm{CdCl}_{2}$ and $\mathrm{ZnCl}_{2}$ solutions, two heavymetal stresses, and analyzed the changes in transcript abundances of $B d P P 2 C$ genes. The expression analysis revealed that $60 \%$ of $B d P P 2 C$ genes shared a similar response pattern between $\mathrm{CdCl}_{2}$ and $\mathrm{ZnCl}_{2}$ treatments. For instance, the expression levels of $B d P P 2 C 2, B d P P 2 C 13$, $B d P P 2 C 30, B d P P 2 C 32, B d P P 2 C 46$ and $B d P P 2 C 47$ were up-regulated after $3 \mathrm{~h}$ and retained relatively high levels for $6 \mathrm{~h}$ when treated with $\mathrm{CdCl}_{2}$ and $\mathrm{ZnCl}_{2}$. 


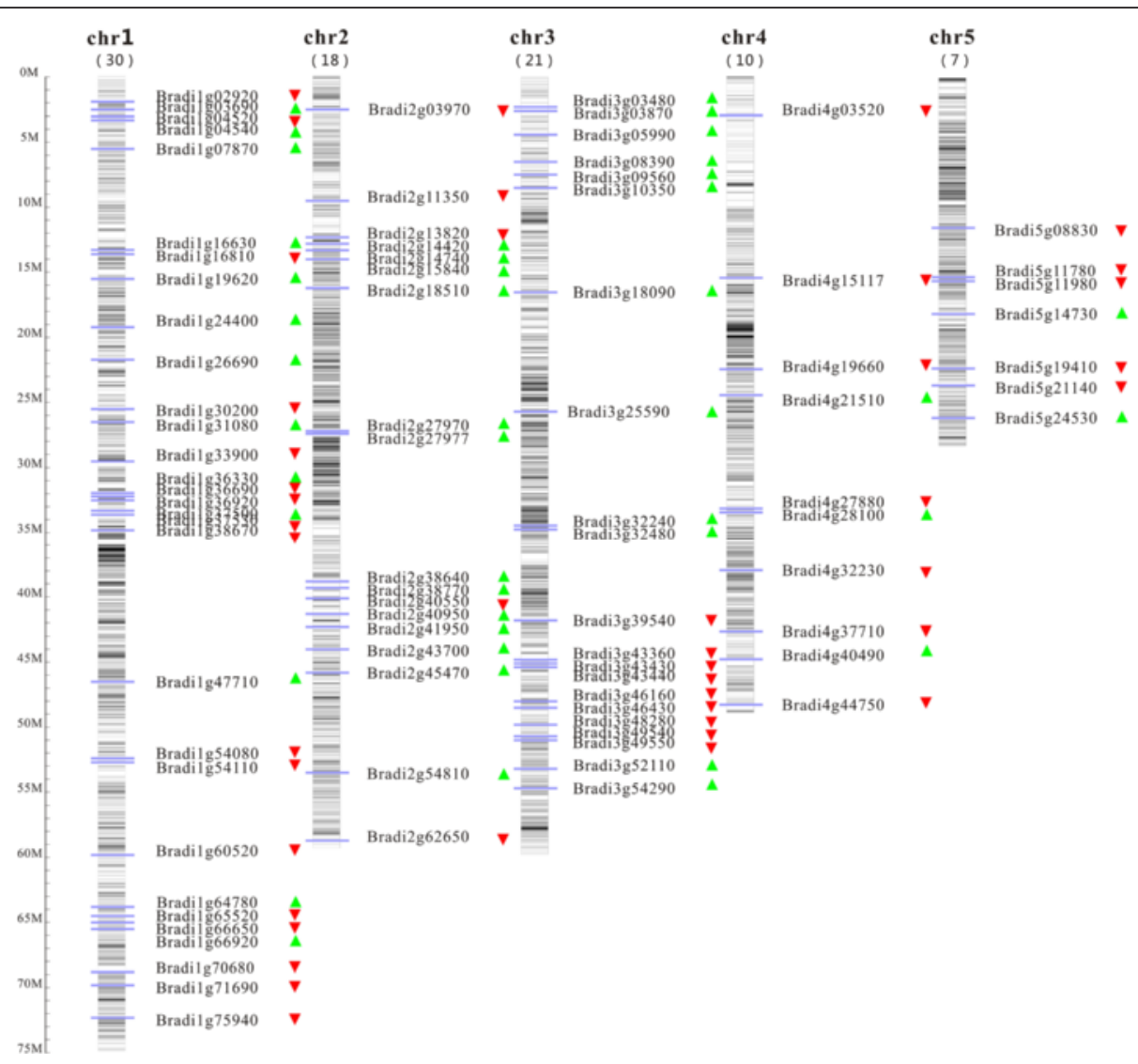

Fig. 4 Genomic distribution of BdPP2C genes on each chromosome. 86 BdPP2C genes were mapped onto specific chromosomes, and the number of BdPP2CS on each chromosome is indicated. The small green or red triangles show the direction of transcription for genes. The deeper color on the chromosomes' region represents the higher density of CpG islands

However, for $B d P P 2 C 37, B d P P 2 C 39, B d P P 2 C 40$, BdPP2C43, BdPP2C55, BdPP2C69, BdPP2C77, BdPP2C82 and $B d P P 2 C 84$, their expression was down-regulated after $3 \mathrm{~h}$ and then up-regulated or returned to basal levels after $6 \mathrm{~h}$.

The previous study has reported that AtPP2CG1 is induced by drought, salt or exogenous ABA treatment and thus positively regulates salt tolerance in Arabidopsis [52]. In this study, BdPP2C32, a homolog of AtPP2CG1, was also found to be strongly responsive to $\mathrm{ABA}$ and abiotic treatments (Fig. 7). Interestingly, $\mathrm{CdCl}_{2}$ and $\mathrm{ZnCl}_{2}$ treatments resulted in an obvious increase in its expression, which indicates that $P P 2 C$ members in the $\mathrm{G}$ group may be related to heavy-metal-resistant pathways in plants. In addition, we also investigated the expression levels of $B d P P 2 C$ s after phytopathogens' infection. $\mathrm{Bd} 21$ seedling was infected with $F$. graminearum (F0968) and two M. griseastrains (Guy11, avirulent ACE1 genotype; PH14, virulent ACE1 genotype). As shown in Fig. 7, many $B d P P 2 C$ s were significantly down-regulated at $4 \mathrm{~h}$ and $12 \mathrm{~h}$ after infection with all three phytopathogens. Only $20 \mathrm{BdPP} 2 \mathrm{Cs}$ were up-regulated by a single phytopathogen within 4-12 h, such as BdPP2C13, BdPP2C14,
$B d P P 2 C 25, B d P P 2 C 42$ and $B d P P 2 C 62$. It has been demonstrated that the PP2C WIN2 (At4g31750), a homolog in Arabidopsis with $B d P P 2 C 13$, can enhance the resistance to phytopathogen $P$. syringae [18]. Consistent with this, the expression of $B d P P 2 C 13$ was remarkably increased after exogenous salicylic treatment and phytopathogen PH14 treatment. The expression profiles of $B d P P 2 C$ genes under different treatments are listed in Additional file 4: Tables S4 and S5. These results indicate that PP2Cs may be involved not only in the regulation of abiotic stresses but also in biotic stresses.

As mentioned above, 22 pairs of paralogous $B d P P 2 C$ genes involved in segmental duplication events have been found, which suggested that chromosome gene duplication may play an important role in the expansion and evolution of the $B d P P 2 C$ gene family. It has been observed that segmentally duplicated genes displayed a greater degree of functional divergence, and they exhibited pseudo-functionalization, neo-functionalization and retention of gene functions [53]. We analyzed the expression pattern of 22 segmentally duplicated gene pairs under various stresses, which showed that 17 pairs of genes had very similar expression pattern, indicating the retention of 


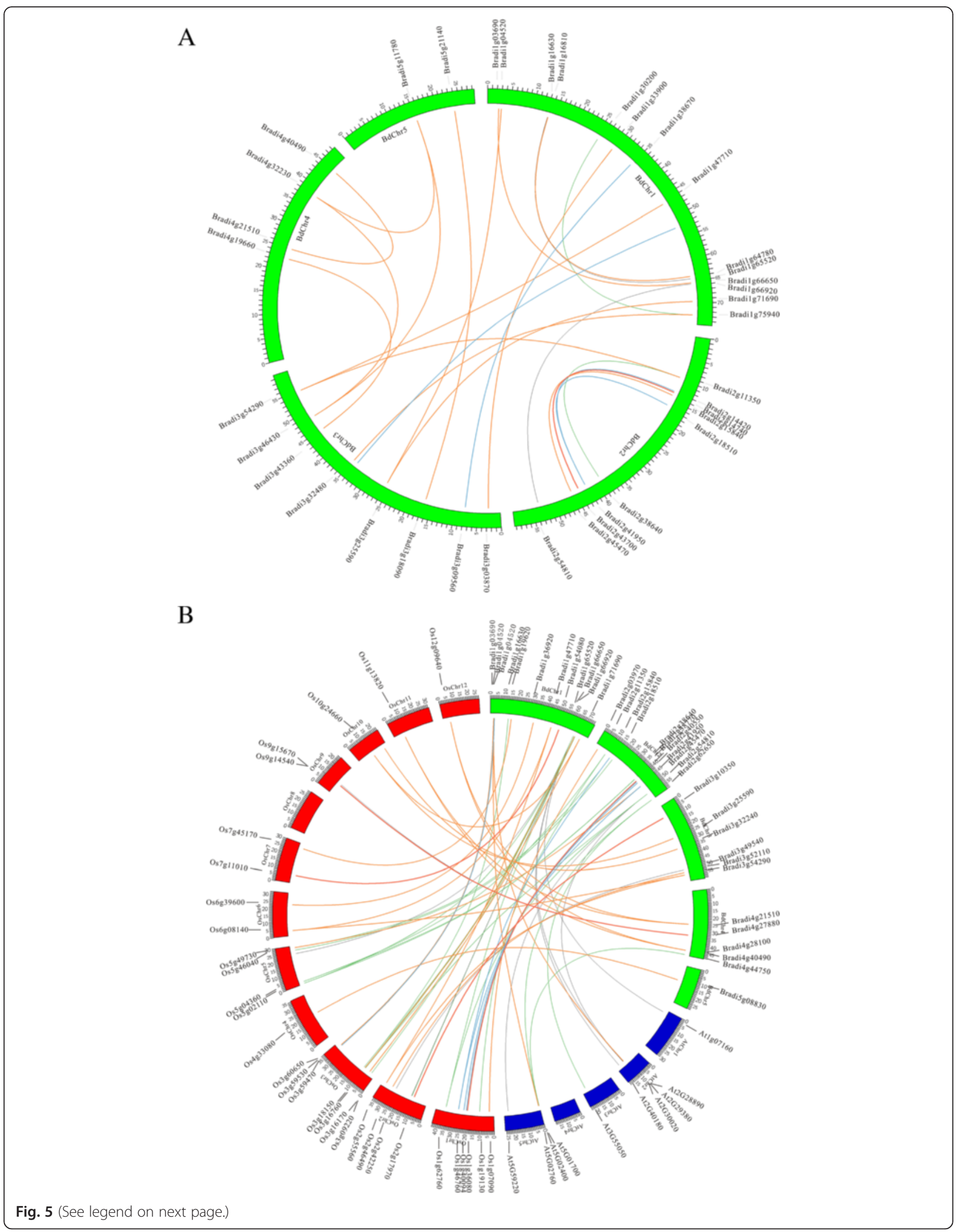


(See figure on previous page.)

Fig. 5 Duplication event analysis of $B d P P 2 C$ genes and comparative synteny analysis among B. distachyon, Arabidopsis and rice. a The data were derived from the Plant Genome Duplication Database, and 22 couples of duplicated BdPP2C genes were anchored to corresponding positions on B. distachyon chromosomes using the CIRCOS program. b Synteny between B. distachyon and Arabidopsis or between B. distachyon and rice was anchored to the corresponding position on specific chromosomes using the CIRCOS program. $B$. distachyon chromosomes are depicted as green segments, and Arabidopsis and rice are shown in blue and red, respectively. The size of chromosomes was consistent with the actual pseudo-chromosome size. Positions are in Mb

their functions (Additional file 5: Figure S5). Most of the duplicated gene pairs were found to have a high amino acid sequence homology and share similar exon/intron structures. Although the diversification of expression occurred between duplicated $P P 2 C$ gene pairs, their functions still were retained. This indicated that diversification of expression may occur after a very short period of time, while function diversification may need more time.

\section{The relevance analysis between cis-acting element and stress-induced $B d P P 2 C$ gene expression}

Cis-acting elements in promoter regions of genes often play key roles in stress responses in plants. For example, ABA-responsive elements (ABREs) are responsive to ABA, drought or salt signals [54]. LTR is involved in low-temperature response and regulation [55]. TCA-element and CGTCA-motif have good correlation with the expression levels after MeJA and SA treatment, respectively [56]. Analyses of cis-acting elements in the promoter regions of the $86 B d P P 2 C$ genes showed that every $B$. distachyon $P P 2 C$ gene carried one or more ABRE, HSE, LTR, TCA-element, and CGTCA-motif in their promoter regions, suggesting the significant relationship between $B d P P 2 C$ s and stress responses (Additional file 6: Figure S4). Among them, $B d P P 2 C$ genes from subgroup A contained the greatest number of ABRE, which is consistent with Arabidopsis, foxtail millet and rice [23, 43]. Further analyses demonstrated that a good correlation existed between the number of the ABREs and the expression of $B d P P 2 C$ in the majority of subgroups after $3 \mathrm{~h}$ or $6 \mathrm{~h} \mathrm{ABA}$ treatment according to the statistical analysis. Similarly, this correlation was also found to exist between the number of ABREs and the expression

Table 3 BdPP2Cs present in segmental duplication in B. distachyon genome

\begin{tabular}{|c|c|c|c|c|c|}
\hline Locus ID & Subgroup & Ka & Ks & $\mathrm{Ka} / \mathrm{Ks}$ & Divergence time (Myr) \\
\hline BdPP2C2/26 & $C$ & 0.52 & 1.00 & 0.52 & 76.9 \\
\hline BdPP2C3/56 & K & 0.68 & 1.55 & 0.438709677 & 119.2 \\
\hline BdPP2C7/24 & $\mathrm{H}$ & 0.10 & 0.76 & 0.131578947 & 58.5 \\
\hline $\mathrm{BdPP} 2 \mathrm{C} 11 / 30$ & $D$ & 0.2 & 0.78 & 0.25641026 & 60 \\
\hline BdPP2C13/50 & $\mathrm{F}$ & 0.11 & 1.34 & 0.082089552 & 103.1 \\
\hline BdPP2C19/53 & $\mathrm{F}$ & 0.28 & 0.75 & 0.373333333 & 57.7 \\
\hline BdPP2C20/69 & G & 0.14 & 1.13 & 0.123893805 & 86.9 \\
\hline BdPP2C25/6 & $B$ & 0.38 & 1.51 & 0.251655629 & 116.2 \\
\hline $\mathrm{BdPP} 2 \mathrm{C} 27 / 47$ & A & 0.35 & 0.76 & 0.460526326 & 58.5 \\
\hline BdPP2C29/56 & K & 0.25 & 1.00 & 0.25 & 76.9 \\
\hline BdPP2C30/58 & $D$ & 0.15 & 0.88 & 0.170454545 & 67.7 \\
\hline BdPP2C32/40 & G & 0.22 & 1.12 & 0.1964285 & 86.2 \\
\hline BdPP2C34/44 & A & 0.25 & 1.41 & 0.177304965 & 108.5 \\
\hline BdPP2C35/45 & $\mathrm{F}$ & 0.15 & 0.66 & 0.227272727 & 50.8 \\
\hline BdPP2C36/46 & A & 0.31 & 0.74 & 0.418918919 & 56.9 \\
\hline BdPP2C37/44 & A & 0.32 & 1.65 & 0.193939394 & 126.9 \\
\hline BdPP2C55/85 & F & 0.12 & 0.84 & 0.142857143 & 64.6 \\
\hline BdPP2C60/72 & E & 0.46 & 1.71 & 0.269005848 & 131.5 \\
\hline BdPP2C64/76 & $\mathrm{H}$ & 0.28 & 1.03 & 0.27184466 & 79.2 \\
\hline BdPP2C69/32 & G & 0.61 & 1.00 & 0.61 & 76.9 \\
\hline BdPP2C73/78 & $B$ & 0.41 & 0.82 & 0.5 & 63.1 \\
\hline BdPP2C76/81 & $\mathrm{H}$ & 0.34 & 1.30 & 0.2615384 & 100 \\
\hline
\end{tabular}




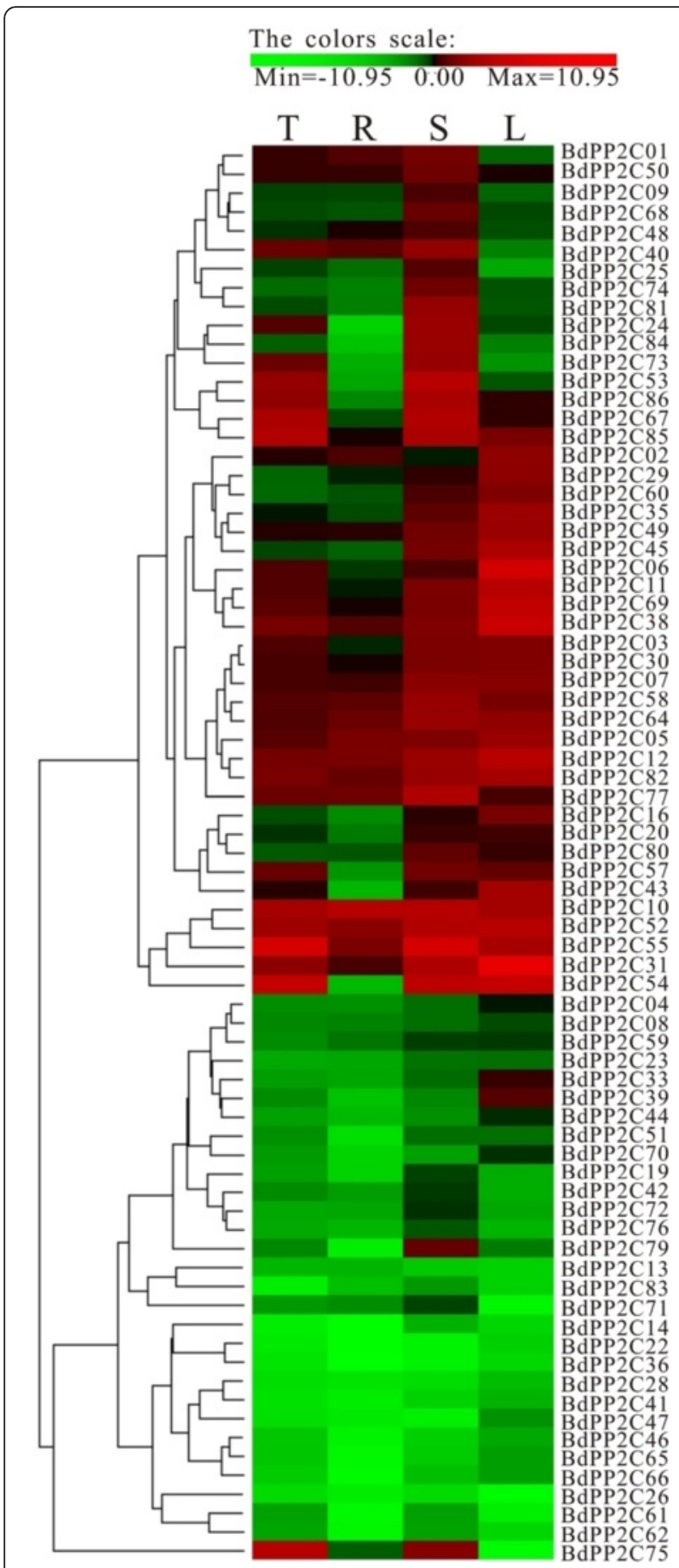

Fig. 6 Expression profiles of $B d P P 2 C$ genes in various tissues. The heat map shows the real-time $\mathrm{qPCR}$ analysis results of $B d P P 2 C$ genes expression in roots, stems, leaves and seedlings. $T$, seedling; $R$, root; $\mathrm{S}$, stem; L, leaf

levels after salt or drought treatment (Additional file 7: Figure S6A-F). This result indicates that $B d P P 2 C$ genes may be involved in drought or salt stress through the ABA-dependent pathway.

\section{Conclusions}

In the present study, beyond a comprehensive analysis of phylogenetic relationships, gene structures, conserved domains, chromosomal locations, gene duplications, ciselements and expression patterns of the $P P 2 C$ gene family was carried out for the first time in a new model monocot i.e., $B$. distachyon, one of the key features of our results was the multiple level approaches to identify BdPP2C members from sequence alignment, motifs and domain analysis to $3 \mathrm{D}$ protein structure confirmation (Fig. 1). Another key feature of our result was the expression patterns of the $B d P P 2 C$ genes indicated that almost all members displayed up-regulation in response to abiotic stresses such as cold, heat, PEG and $\mathrm{NaCl}$ treatments, but down-regulation to biotic stresses such as Ph14, Guy11 and F0968 infection (Fig. 7).

\section{Methods}

Database searching and sequence retrieval

To identify $P P 2 C$ candidates in $B$. distachyon, the InterPro domain "IPR001932" was used to search the Plaza2.5 database (http://bioinformatics.psb.ugent.be/plaza/versions/ plaza_v2_5/). The resulting protein sequences were manually examined with Pfam (http://pfam.sanger.ac.uk/search) and SMART (http://smart.embl-heidelberg.de/) to confirm the presence of the PP2C domains [57]. Arabidopsis PP2C protein sequences were downloaded from the TAIR database (https://www.arabidopsis.org). PP2C protein sequences of rice were downloaded from the Rice Genome Annotation Project Database (https://rice.plantbiology. msu.edu/). The molecular weight (MW) and theoretical isoelectric point ( $\mathrm{pI}$ ) of the PP2C candidates were calculated using the Compute $\mathrm{pI} / \mathrm{MW}$ tool in the ExPASy server (http://web.expasy.org/compute). The WoLF PSORT program (http://expasy.org/resources/search/keywords:subcellular\%20location) was used to predict protein subcellular localization. The prediction of protein tertiary structure was done with the Phyre2 tool (http:// www.sbg.bio.ic.ac.uk/phyre2/) [58].

\section{Phylogenetic analysis, exon/intron analysis and domain analysis}

For phylogenetic tree construction, amino acid sequences of PP2C domains in B. distachyon, Arabidopsis and rice were aligned using the Clustal W2 program [59], and then phylogenetic trees were constructed using the ML method based on the JTT matrix-based model with MEGA 6.0 [60]. The data for the phylogenetic tree were deposited in Treebase Web (Accession URL: http://purl.org/phylo/ treebase/phylows/study/TB2:S17847You). The exon/intron structures of the $P P 2 C$ gene candidates were examined using the online Gene Structure Display Server (GSDS: http://gsds.cbi.pku.edu.ch) based on their corresponding genomic sequences [61]. The conserved 


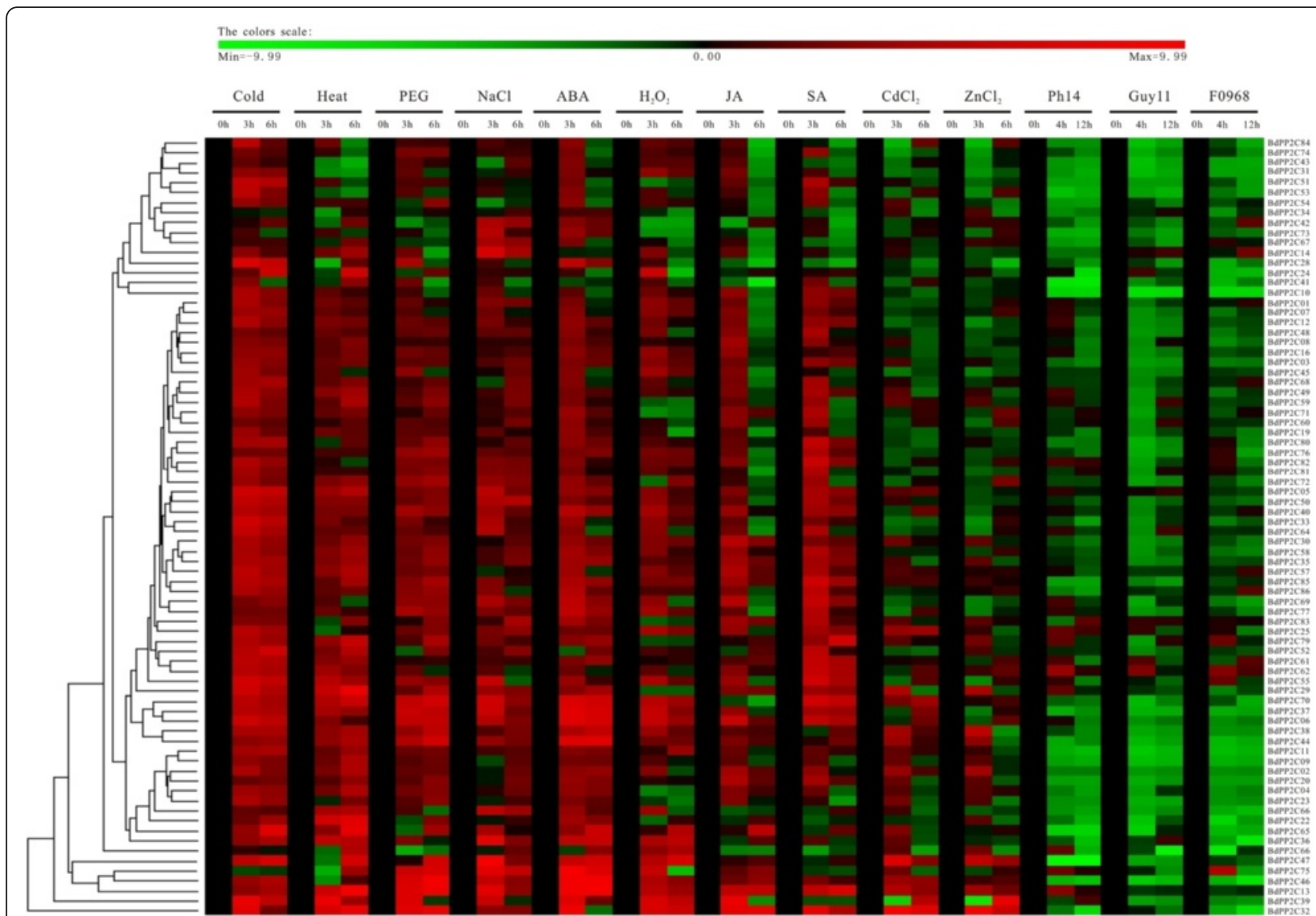

Fig. 7 Expression profiles of $B d P P 2 C$ genes under biotic/abiotic stresses and phytohormone treatments. Clustering of all $B d P P 2 C$ genes according to their expression profiles under biotic/abiotic stresses and phytohormone treatments. The expression levels of genes are presented using fold-change values transformed to $\log _{2}$ format compared with the control. The color bar represents fold-change values: green, representing down-regulation $\left(\log _{2} \leq-1\right)$; red, representing up-regulation $\left(\log _{2} \geq 1\right)$

domains were predicted using the InterPro database (http://www.ebi.ac.uk/interpro/).

\section{Chromosomal locations and synteny analysis}

The chromosomal locations of PP2C genes were derived from the Plaza2.5 database. For synteny analysis, we investigated gene duplication events of $P P 2 C$ genes in the $B$. distachyon genome and among $B$. distachyon, Arabidopsis and rice genomes from the Plant Genome Duplication Database (http://chibba.agtec.uga.edu/duplication/index/ locus) [62]. Then the synteny blocks were illustrated with CIRCOS software (http://circos.ca/software/download/ circos) [63].

\section{Plant materials and hormone, abiotic-and biotic-stress treatments}

B. distachyon $\mathrm{Bd} 21$ was cultured in $1 / 4$ Hogland's solution at $25{ }^{\circ} \mathrm{C} / 22{ }^{\circ} \mathrm{C}$ (day/night) with supplemental lighting for two weeks. Young roots, stems and leaves were harvested for tissue-specific expression analysis. Before the stress treatments, the 2-week-old seedlings were soaked in deionized water for two hours. For abiotic stress treatment, the seedlings were incubated in a solution containing $20 \%$ PEG, $200 \mathrm{mM} \mathrm{NaCl}, 10 \mathrm{mM} \mathrm{H}_{2} \mathrm{O}_{2}$ or $100 \mu \mathrm{M}$ $\mathrm{ZnCl}_{2} / \mathrm{CdCl}_{2}$. The cold or heat treatment was applied by incubating seedlings in a beaker containing water pretreated to $4{ }^{\circ} \mathrm{C}$ or $42{ }^{\circ} \mathrm{C}$, respectively. For phytohormone analysis, the seedlings were cultured in deionized water containing $100 \mu \mathrm{M}$ MeJA, $100 \mu \mathrm{M}$ ABA or $1 \mathrm{mM}$ SA. Whole seedlings were collected from each stress treatment after $0 \mathrm{~h}, 3 \mathrm{~h}$ and $6 \mathrm{~h}$ exposure. Phytopathogen treatment was conducted by spraying 2-week-old seedlings with $\mathrm{Fu}$ sarium graminearum (F0968) or two Magnaporthe grisea strains (Guy11, avirulent ACE1 genotype; PH14, virulent ACE1genotype) for $4 \mathrm{~h}$ and $12 \mathrm{~h}$ according to the previous report [55]. These tissues and seedlings were immediately frozen in liquid nitrogen and stored at $-80^{\circ} \mathrm{C}$ until used.

\section{Quantitative reverse transcription PCR (qRT-PCR) and cluster analysis}

The total RNA was extracted from plant tissues and seedlings using RNAiso Plus (Takara, Dalian, China) 
according to the manufacturer's instructions. About 1$2 \mu \mathrm{g}$ total RNA was used to perform reverse transcription using the PrimeScript ${ }^{\text {tm }}$ RT reagent kit with gDNA Eraser $^{\text {rx }}$ to remove genomic DNA contamination (Takara, Dalian, China). The resulting cDNA was diluted 20-fold with sterile distilled water. Quantitative PCR (qPCR) was performed in an ABI StepOne Real-Time Cycler (Applied Biosystems, USA) using $10 \mu \mathrm{L}$ reaction volume containing $1 \mu \mathrm{L}$ cDNA, $0.4 \mu \mathrm{L}$ forward primer $(10 \mu \mathrm{M}), 0.4 \mu \mathrm{L}$ reverse primer $(10 \mu \mathrm{M}), 0.2 \mu \mathrm{L}$ ROX and $5 \mu \mathrm{L}$ SYBR Premix Ex Taq ${ }^{\mathrm{T}} \mathrm{II}$ (Takara, Dalian, China). The following condition was used: pre-denaturation at $95{ }^{\circ} \mathrm{C}$ for $30 \mathrm{~s}, 40$ cycles of amplification at $95{ }^{\circ} \mathrm{C}$ for $5 \mathrm{~s}$ and $60{ }^{\circ} \mathrm{C}$ for $30 \mathrm{~s}$. Gene-specific primers was designed using the Primer5 program and their specificity was checked by blastN. The primers used for qPCR are listed in Additional file 4: Table S2 and actin was used as an internal control $[56,64,65]$. The data reported in this study were calculated based on three biological replicates. In addition, the expression profiles were calculated using the $-\Delta \Delta \mathrm{CT}$ values $\left[-\Delta \Delta \mathrm{CT}=\left(\mathrm{CT}\right.\right.$ control $_{\text {gene }}-\mathrm{CT}$ Tcontrol $\left._{\text {actin }}\right)$ $-\left(\right.$ CTtreated $_{\text {gene }}-$ CTtreated $\left.\left._{\text {actin }}\right)\right]$, and obtained by PermutMatrixEN version 1.9.3 software, and then shown by a green-red gradient. The data were statistically analyzed using OriginPro 7.5. The differentially expressed genes were defined as those showing up-or downregulation greater than twofold with $p$-value less than 0.05. All qPCR data were submitted to the NCBI GEO dataset with accession number GSE70366.

\section{Promoter analysis}

The 1500 bp promoter sequences of $B d P P 2 C$ genes were obtained from the Plaza2.5 database. The cis-acting regulatory elements in these promoter sequences were analyzed using the PLANT CARE program (http:// bioinformatics.psb.ugent.be/webtools/plantcare/html/) [66].

\section{Availability of supporting data}

The qRT-PCR data supporting the genes expression results of this study are available in the NCBI GEO database with accession number GSE70366. The supporting tables for phylogenetic tree were deposited in Treebase Web (URL: http://purl.org/phylo/treebase/phylows/ study/TB2:S17847You).

\section{Additional files}

Additional file 1: Figure S1. The amino acid alignment of $86 \mathrm{BdPP} 2 \mathrm{C}$ domains in B. distachyon. (TIF $9808 \mathrm{~kb}$ )

Additional file 2: Figure S2. The predicted 3-D structure of $86 \mathrm{BdPP} 2 \mathrm{C}$ domains in B. distachyon. (TIF $8875 \mathrm{~kb}$ )

Additional file 3: Figure S3. The predicted tertiary structure of 86 BdPP2C domains in B. distachyon. (TIF $7796 \mathrm{~kb}$ )
Additional file 4: Table S1. The amino acid sequences of $P P 2 C$ genes in B. distachyon, Arabidopsis and rice. Table S2. The primers used for real-time $\mathrm{qPCR}$ in this study. Table $\mathbf{S 3}$. The expression profiles of $B d P P 2 C s$ in various tissues. Table S4. The expression profiles of BdPP2Cs under abiotic or hormone treatment. Table S5. The expression profiles of BdPP2Cs under biotic stress. Table S6. The orthologous PP2C gene pairs between $B$. distachyon and Arabidopsis or B. distachyonand rice. (DOCX $308 \mathrm{~kb})$

Additional file 5: Figure S5. Expression pattern of duplication BdPP2C genes under biotic/abiotic stresses and phytohormone treatments. (TIF $9276 \mathrm{~kb}$ )

Additional file 6: Figure S4. Cis-acting elements analysis in promoter sequences of 86 BdPP2C genes. (TIF $9862 \mathrm{~kb}$ )

Additional file 7: Figure S6. The correlation analysis between cis-acting elements and stress-induced expression of BdPP2C genes. (A) The correlation analysis between $A B R E$ and $A B A$-induced expression of $B d P P 2 C$ genes. The samples were treated with $A B A$ for 3 h. (B) The correlation analysis between $A B R E$ and $A B A$-induced expression of BdPP2C genes. The samples were treated with ABA for $6 \mathrm{~h}$. (C) The correlation analysis between $\mathrm{ABRE}$ and $\mathrm{NaCl}$-induced expression of $\mathrm{BdPP} 2 \mathrm{C}$ genes. The samples were treated with $\mathrm{NaCl}$ for $3 \mathrm{~h}$. (D) The correlation analysis between ABRE and $\mathrm{NaCl}$-induced expression of BdPP2C genes. The samples were treated with $\mathrm{NaCl}$ for $6 \mathrm{~h}$. (E) The correlation analysis between $A B R E$ and PEG-induced expression of BdPP2C genes. The samples were treated with PEG for $3 \mathrm{~h}$. (F) The correlation analysis between ABRE and PEG-induced expression of BdPP2C genes. The samples were treated with PEG for $6 \mathrm{~h}$. (TIF $6349 \mathrm{~kb}$ )

\section{Abbreviations}

ABRE: ABA-responsive element; AMPK: AMP-activated protein kinase; CCaMK: $\mathrm{Ca}^{2+} /$ calmodulins-dependent protein kinases; CDPK: calcium dependentprotein kinases; CNB: cyclic nucleotide-binding domain; DSPTPS: dual-specificity phosphatases; FHA: forkhead associated domain; KAPP: kinase associated protein phosphatase; MAPK: mitogen-activated protein kinases; MW: molecular weight; Mya: million years ago; PP1: protein phosphatase 1; PP4: protein phosphatase 4; PP5: protein phosphatase 5; PP6: protein phosphatase 6; PP7: protein phosphatase 7; PP2As: protein phosphatases 2A; PP2Bs: protein phosphatases 2B; PP2Cs: protein phosphatases 2C; Pi: isoelectric point; PPM: phosphoprotein metallophosphatase; PPP: phosphor-protein phosphatase; PTPs: protein tyrosine phosphatases; qRT-PCR: quantitative reverse transcription-PCR: RLK: receptor like protein kinase; SnRK2: Nonfermenting1-related protein kinases2; S-TKc: Ser/thr kinase catalytic domain.

\section{Competing interests}

The authors declare that they have no competing interests.

\section{Authors' contributions}

ZC and JC conceived of the study. JC, ZC, MJ and PL performed the bioinformatic analysis and carried out the experiments. JC and ZC wrote the manuscript. All authors read and approved the final manuscript.

\section{Acknowledgments}

This work was supported by Grant from Shanghai Landscaping Administrative Bureau (grant Nos.G152423) and the grant from Science and Technology Commission of Shanghai Municipality (14DZ2260400). We are grateful to Dr. Feng Wen of Shanghai Chenshan Botanical Garden for kindly providing some consultancy.

Received: 23 July 2015 Accepted: 24 February 2016

Published online: 03 March 2016

\section{References}

1. Luan S. Protein phosphatases and signaling cascades in higher plants. Trends Plant Sci. 1998:3(7):271-5.

2. Mizoguchi T, Ichimura K, Shinozaki K. Environmental stress response in plants: the role of mitogen-activated protein kinases. Trends Biotechnol. 1997;15:15-9. 
3. Boudsocq $M$, Barbier-Brygoo $H$, Lauriere $C$. Identification of nine sucrose nonfermenting 1-related protein kinases 2 activated by hyperosmotic and saline stresses in Arabidopsis thaliana. J Biol Chem. 2004;279:41758-66.

4. Ma S, Wu W. AtCPK23 functions in Arabidopsis responses to drought and salt stresses. Plant Mol Biol. 2007;65:511-8.

5. Franz S, Ehlert B, Liese A, Kurth J, Cazale AC, Romeis T. Calcium-dependent protein kinase CPK21 functions in abiotic stress response in Arabidopsis thaliana. Mol Plant. 2011;4(1):83-96.

6. Kerk D, Templeton G, Moorhead GBG. Evolutionary radiation pattern of novel protein phosphatases revealed by analysis of protein data from the completely sequenced genomes of humans, green algae and higher plants. Plant Physiol. 2008;146:351-67.

7. Cohen P. The structure and regulation of protein phosphatases. Annu Rev Biochem. 1989;58:453-508.

8. Brody MS, Stewart V, Price CW. Bypass suppression analysis maps the signalling pathway within a multidomain protein. Mol Microbiol. 2009;72:1221-34.

9. Gonza A, Ruiz A, Serrano R, Arin J, Casamayor A. Transcriptional profiling of the protein phosphatase $2 \mathrm{C}$ family in yeast provides insights into the unique functional roles of Ptc1. J Biol Chem. 2006;281:35057-69.

10. Corton JM, Gillespie JG, Hardie DG. Role of the AMP-activated protein kinase in the cellular stress response. Curr Biol. 1994;4:315-24.

11. Hirayama T, Umezawa T. The PP2C-SnRK2 complex: the central regulator of an abscisic acid signaling pathway. Plant Signal Behave. 2010;5(2):160-3.

12. Schweighofer A, Kazanaviciute $V$, Scheikl E, Teige M, Doczi R, Hirt H, et al. The PP2C-type phosphatase AP2C1, which negatively regulates MPK4 and MPK6, modulates innate immunity, jasmonic acid, and ethylene levels in Arabidopsis. Plant Cell. 2007;19:2213-24.

13. Gagne JM, Clark SE. The Arabidopsis stem cell factor POLTERGEIST is membrane localized and phospholipid stimulated. Plant Cell. 2010;22:729-43.

14. Song SK, Hofhuis H, Lee MM, Clark SE. Key divisions in the early Arabidopsis embryo require POL and PLL1 phosphatases to establish the root stem cell organizer and vascular axis. Dev Cell. 2008;15:98-109.

15. Servet C, Benhamed M, Latrasse D, Kim W, Delarue M, Zhou DX. Characterization of a phosphatase $2 \mathrm{C}$ protein as an interacting partner of the histone acetyl transferase GCN5 in Arabidopsis. Biochim Biophys Acta. 2008;1779:376-82.

16. Benhamed M, Bertrand C, Servet C, Zhou DX. Arabidopsis GCN5, HD1, and TAF1/HAF2 interact to regulate histone acetylation required for lightresponsive gene expression. Plant Cell. 2006;18:2893-903.

17. Galbiati M, Simoni L, Pavesi G, Cominelli E, Francia P, Vavasseur A, et al. Gene trap lines identify Arabidopsis genes expressed in stomatal guard cells. Plant J. 2008;53:750-62.

18. Lee MW, Jelenska J, Greenberg JT. Arabidopsis proteins important for modulating defense responses to Pseudomonas syringae that secrete HopW1-1. Plant J. 2008;54:452-65.

19. Gomez-Gomez L, Bauer Z, Boller T. Both the extracellular leucine-rich repeat domain and the kinase activity of FSL2 are required for flagellin binding and signaling in Arabidopsis. Plant Cell. 2001;13:1155-63.

20. Stone JM, Collinge MA, Smith RD, Horn MA, Walker JC. Interaction of a protein phosphatase with an Arabidopsis serine-threonine receptor kinase. Science. 1994;266:793-5.

21. Kerk D, Bulgrien J, Smith DW, Barsam B, Veretnik S, Gribskov M. The complement of protein phosphatase catalytic subunits encoded in the genome of Arabidopsis. Plant Physiol. 2002;129:908-25.

22. Schweighofer A, Hirt H, Meskiene I. Plant PP2C phosphatases: emerging functions in stress signaling. Trends Plant Sci. 2004;9:236-43.

23. Xue T, Wang D, Zhang S, Ehlting J, Ni F, Jakab S, et al. Genome-wide and expression analysis of protein phosphatase $2 \mathrm{C}$ in rice and Arabidopsis. BMC Genomics. 2008;9:550.

24. Singh A, Giri J, Kapoor S, Tyagi AK, Pandey GK. Protein phosphatase complement in rice: genome-wide identification and transcriptional analysis under abiotic stress conditions and reproductive development. BMC Genomics. 2010;11:435.

25. The International Brachypodium Initiative. Genome sequencing and analysis of the model grass Brachypodium distachyon. Nature. 2010;463:763-8.

26. Fuchs S, Grill E, Meskiene I, Schweighpfer A. Type 2 C protein phosphatases in plants. FEBS J. 2013:280:681-93.

27. Bork E, Brown NP, Hegyi H, Sohultz J. The protein phosphatase 2C(PP2C) super family:detection of bacterial homologues. Protein Sci. 1996;5(7): 1421-5.

28. Shi YG. Serine/Threonine phosphatases: mechanism through structure. Cell. 2009;139:468-84.
29. Lurin C, Andres C, Aubourg S, Bellaoui M, Bitton F, Bruyere C, et al. Genome-wide analysis of Arabidopsis pentatricopeptide repeat proteins reveals their essential role in organdie biogenesis. Plant Cell. 2004;16(8): 2089-103.

30. Jaill M, Tyagi AK, Khurana JP. Genome-wide analysis, evolutionary expansion, and expression of early auxin-responsive SAUR gene family in rice (oryza sativa). Genomits. 2006;88(3):360-71.

31. Lcohamy A, Boudet I, Aubourg S, Kreis M. Introns in, introns out in plant gene families:a genomic approach of the dynamics of gene structure. J Struct Funct Genomics. 2003;3(14):111-6.

32. Cannon SB, Mitra A, Baumgarten A, Young ND, May G. The roles of segmental and tandem gene duplication in the evolution of large gene families in Arabidopsis thaliana. BMC Plant Biol. 2004:4:10.

33. Cheung J, Estivill X, Khaja R, Macdonald JR, Lau K, Taui LC, et al. Genomewide detection of segmental duplications and potential assembly errors in the human genome sequence. Genome Biol. 2003;4:R25.

34. Lynch M, Conery JS. The evolutionary fate and consequences of duplicate genes. Science. 2000;290:1151-5.

35. Blanc G, Wolfe KH. Widespread paleopolyploidy in model plant species inferred from age distributions of duplicate genes. Plant Cell. 2004;16:1667-78.

36. Gaut BS. Evolutionary dynamics of grass genomes. New Phytol. 2002;154: $15-28$.

37. Blanc $\mathrm{G}$, Wolfe $\mathrm{KH}$. Functional divergence of duplicated genes formed by polyploidy during Arabidopsis evolution. Plant Cell. 2004;16:1679-91.

38. Altenhoff A, Dessimoz C. Phylogenetic and functional assessment of orthologs inference projects and methods. PLos Comput Biol. 2009;5(1), e1000262.

39. Makova KD, Li WH. Divergence in the spatial pattern of gene expression between human duplicate genes. Genome Res. 2003;13:1638-45.

40. Li WH, Yang J, Gu X. Expression divergence between duplicate genes. Trends Genet. 2005;21:602-7.

41. Zhang Z, Gu J, Gu X. How much expression divergence after yeast gene duplication could be explained by regulatory motif evolution? Trends Genet. 2004:20:403-7.

42. Fujita Y, Fujita M, Shinozaki K, Yamaguchi-Shinozaki K. ABA-mediated transcriptional regulation in response to osmotic stress in plants. J Plant Res. 2011;124:509-25.

43. Min D, Xue F, Ma Y, Chen M, Xu Z, Li L, et al. Characteristics of PP2C family in Fixtail Millet (Setaria italica). Acta Agronomica Sinica. 2013;39(12):2135-44.

44. Merlot S, Gosti F, Guerrier D, Vavasseur A, Giraudat J. The ABI1 and ABI2 protein phosphatases $2 \mathrm{C}$ act in a negative feedback regulatory loop of the abscisic acid signalling pathway. Plant J. 2001;25:295-303.

45. Che I, Michard E, Platet N, Mouline K, Alcon C, Sentenac H, et al. Physical and functional interaction of the Arabidopsis $\mathrm{K}^{+}$channel AKT2 and phosphatase AtPP2CA. Plant Cell. 2002;14:1133-46.

46. Saez A, Apostolova N, Gonzalez-Guzman M, Gonzalez-Garcia MP, Nicolas C, Lorenzo O, et al. Gain-of-function and loss-of-function phenotypes of the protein phosphatase $2 \mathrm{CHAB} 1$ reveal its role as a negative regulator of abscisic acid signalling. Plant J. 2004;37:354-69.

47. Yoshida T, Nishimura N, Kitahata N, Kuromori T, Ito T, Asami T, et al. ABA-hypersensitive germination3 encodes a protein phosphatase $2 \mathrm{C}$ (AtPP2CA) that strongly regulates abscisic acid signaling during germination among Arabidopsis protein phosphatase 2Cs. Plant Physiol. 2006;140: 115-26.

48. Umezawa T, Sugiyama N, Mizoguchi M, Hayashi S, Myouga F, YamaguchiShinozaki K, et al. Type $2 \mathrm{C}$ protein phosphatases directly regulate abscisic acid-activated protein kinases in Arabidopsis. Proc Natl Acad Sci U S A. 2009; 106:17588-93

49. Zhang J, Li X, He Z, Zhao X, Wang Q, Zhou B, et al. Molecular character of a phosphatase $2 \mathrm{C}$ (PP2C) gene relation to stress tolerance in Arabidopsis thaliana. Mol Biol Rep. 2013:40:2633-44

50. Shinozaki K, Yamaguchi-Shinozaki K. Molecular responses to dehydration and low temperature: differences and cross-talk between two stress signaling pathways. Curr Opin Plant Biol. 2000;3(3):217-23.

51. Knight $H$, Knight MR. Abiotic stress signaling pathways: specificity and crosstalk. Trends Plant Sci. 2001;6(6):262-7.

52. Liu X, Zhu Y, Zhai H, Cai H, Ji W, Luo X, et al. AtPP2CG1, a protein phosphatase $2 \mathrm{C}$, positively regulates salt tolerance of Arabidopsis in abscisic acid-dependent manner. Biochem Bioph Res Co. 2012;422:710-5.

53. Prince VE, Pickett FB. Splitting of pairs: the diverging fates of duplicated genes. Nat Rev Genet. 2002;3:827-37. 
54. Li W, Cui X, Meng Z, Huang X, Xie Q, Wu H, et al. Transcriptional regulation of Arabidopsis MIR168a and ARGONAUTE1 homeostasis in ABA and abiotic stress responses. Plant Physiol. 2012;158:1279-92.

55. Maestrini P, Cavallini A, Rizzo M, Giordani T, Bernardi R, Durante M, et al. Isolation and expression analysis of low temperature induced genes in white poplar (Populus alba). J Plant Physiol. 2009;166:1544-56.

56. Wen F, Zhu H, Li P, Jiang M, Mao W, Ong C, et al. Genome-wide evolutionary characterization and expression analyses of WRKY family genes in Brachypodium distachyon. DNA Res. 2014;21(3):327-39.

57. Letunic I, Doerks T, Bork P. SMART 7: recent updates to the protein domain annotation resource. Nucleic Acids Res. 2012;40:302-5.

58. Kelley LA, Sternberg MJE. A case study using the phyre server. Nat Protoc. 2009:4:363-71.

59. Larkin MA, Blackshields G, Brown NP, Chenna R, McGettigan PA, McWilliam $\mathrm{H}$, et al. Clustal W and Clustal X version 2.0. Bioinformatics. 2007;23(21): 2947-8.

60. Tamura K, Stecher G, Peterson D, Filipski A, Kumar S. MEGA 6: molecular evolutionary genetics analysisversion 6.0. Mol Biol Evol. 2013;30:2725-9.

61. Guo AY, Zhu QH, Chen X, Luo JC. GSDS: a gene structure display server. Yi Chuan. 2007;29(8):1023-6.

62. Lee $T H$, Tang $H$, Wang $X$, Paterson AH. PGDD: a database of gene and genome duplication in plants. Nucleic Acids Res. 2013;41(1):1152-8.

63. Krzywinski M, Schein J, Birol I, Connors J, Gascoyne R, Horsman D, et al. Circos: an information aesthetic for comparative genomics. Genome Res. 2009;19:1639-45.

64. Jiang M, Wen F, Cao JM, Li P, She J, Chu Z. Genome-wide exploration of the molecular evolution and regulatory network of mitogen-activated protein kinase cascades upon multiple stresses in Brachypodium distachyon. BMC Genomics. 2015;16:227.

65. Liu X, Chu Z. Genome-wide evolutionary characterization and analysis of bZIP transcription factors and their expression profiles in response to multiple abiotic stresses in Brachypodium distachyon. BMC Genomics. 2015;16:228.

66. Leaeor M, Dehais P, Thijs G, Marchal K, Morcau Y, Rouze P, et al. PlantCARE, a database of plant cis-acting regulatory elements and a portal to tools for in silico analysis of promoter sequences. Nucleic Acids Res. 2002;30(1):325-7.

\section{Submit your next manuscript to BioMed Central and we will help you at every step:}

- We accept pre-submission inquiries

- Our selector tool helps you to find the most relevant journal

- We provide round the clock customer support

- Convenient online submission

- Thorough peer review

- Inclusion in PubMed and all major indexing services

- Maximum visibility for your research

Submit your manuscript at www.biomedcentral.com/submit 